\title{
Modulation Aware Connection Admission Control ANd Uplink Scheduling Algorithm For WIMAX NETWORKS
}

\author{
Shwetha $\mathrm{D}^{1}$, Mohan Kumar $\mathrm{N} \mathrm{M}^{2}$ and Devaraju $\mathrm{J} \mathrm{T}^{2}$ \\ ${ }^{1}$ Department of Electronics, Tumkur University, Tumkur, India \\ ${ }^{2}$ Department of Electronic Science, Bangalore University, Bangalore, India
}

\begin{abstract}
Mobile WiMAX standard defines air interface for mobile broadband wireless access (BWA) systems and aimed to provide high data rate with seamless mobility while maintaining the quality of service $(Q o S)$. However, QoS of real-time applications depends on the link quality, which necessitate the channel-aware connection admission control (CAC) and scheduling schemes. In this paper Modulation aware CAC scheme and base station (BS) uplink scheduling algorithm are proposed to improve the system capacity and QoS performance respectively. The proposed CAC scheme aims to increase the admission of higher priority real time services by degrading the bandwidth of admitted lower priority connections on the basis of their channel status without deteriorating their QoS performance much. The performances of proposed algorithms are evaluated through simulation by considering the metrics like throughput, delay and number of connections admitted.
\end{abstract}

\section{KEYWORDS}

Mobile WiMAX, Broadband Wireless Access, Connection Admission Control, channel aware and Uplink scheduling

\section{INTRODUCTION}

Quality of service (QoS) guarantee is one of the important requirements in today's communication era which is due to the stringent quality requirements of multimedia services. In communication systems QoS is provided through connection admission control (CAC), traffic policing and scheduling. These traffic control mechanisms enable a balance between the utilization of the network resources and the QoS provisioning. This tradeoff between utilization and QoS is of fundamental importance in WiMAX networks [1], which aggregate different types of traffic in a limited resources architecture.

The scheduling mechanism aims at guaranteeing the bandwidth required by the subscriber stations (SS) as well as enabling the efficient wireless link usage. In a WiMAX network, the downlink scheduling requires a single scheduler at the base station (BS), whereas the uplink scheduling needs two components, one of them at the BS and the second one at the SS. The BS scheduler allocates bandwidth for the SSs and the SS scheduler determines which packets will be sent in the received transmission opportunities. In WiMAX, scheduling will be made on the basis of scheduling classes (service types) defined. WiMAX defines five scheduling classes, unsolicited grant service (UGS), extended real time polling service (ertPS), real time polling service (rtPS), non real time polling service (nrtPS) and best effort (BE) services. The 802.16e standard defines 
uplink scheduling only for UGS services and for all other services scheduling is kept as an open issue.

In WiMAX network, before an SS initiates a new connection, it must make a request to the BS. The task of CAC is to accept or reject the arriving requests for a connection in order to maximize the bandwidth utilization, by accepting as many connections as possible, while maintaining the QoS level of admitted connections. If the $\mathrm{CAC}$ admits too few connections, it results in wastage of system resources. On the other hand, if CAC admits too many connections, admitted connections contend for resources and scheduler will not be able to provide QoS. The CAC only guarantees the bandwidth resources for an admitted connection, the actual allocation of bandwidth resources for that admitted connection will be made by the BS uplink scheduler based on the availability of bandwidth.

Awareness of channel status in scheduling and CAC improves the system performance, as there is a possibility of deterioration of QoS of connections as the channel quality varies [2]. Hence in this paper, modulation aware BS scheduling and CAC schemes are proposed to improve the network utilization and QoS. The rest of this paper is organized as follows. Section 2 outlines the related work in the literature. In Sections 3 and 4, proposed CAC and scheduling algorithms are presented respectively. Section 5 discusses the simulation results followed by conclusion in Section 6.

\section{RELATED WORK}

As the CAC and scheduling are the major issues in providing QoS for WiMAX network, many researchers have proposed the algorithms for CAC and scheduling. Authors of [3-4] proposed CAC algorithms which provide the highest priority for UGS flows and maximizes the bandwidth utilization, in [3] bandwidth borrowing and degradation method is employed and in [4] handoff connections are given higher priority. In order to avoid the QoS degradation authors of [5] have proposed a statistical CAC mechanism which considers the traffic variability and overflow. Authors of [6-8] have proposed CAC algorithms which are based on bandwidth estimation of connections, in which [8] considers the shadow cluster concept to estimate the resources. Authors of [9-10] proposed bandwidth degradation scheme for CAC, in which [9] employs interference ratio (IR) as the criteria of degradation and [10] uses the concept of adaptive bandwidth degradation. Scheduling and CAC schemes are proposed in [11-12], among which [11] is concerned with real-time video applications and [12] considers bandwidth polling overhead incurred by services being requested and fairness in resource allocation. Y. Ge et.al [13] proposed a CAC scheme for adaptive multimedia services. Authors of [14-16] have proposed CAC scheme along with packet scheduling algorithm. In [14] dual partition (DP) of the bandwidth for CAC and priority earliest deadline for packet scheduling is proposed.

\section{Proposed Modulation Aware Connection Admission Control MEChANISM}

In this paper modulation aware connection admission control (CAC) algorithm and BS uplink scheduling algorithms are proposed to improve the system capacity in terms of number of connections admitted along with enhancement in QoS.

In WiMAX standard, adaptive modulation and coding (AMC) technique is used to enhance the system performance in varying channel condition. To aid the AMC process the SS feedbacks the channel state information (CSI) to the BS. After receiving the CSI, the BS decides the appropriate 
modulation and coding scheme (MCS) for that SS, i.e., based on CSI it selects one of the modulation schemes among 64-QAM, 16QAM and QPSK modulation. The SS uses this MCS to transmit its data burst [17-18]. This work makes an attempt to utilize channel information to design the proposed $\mathrm{CAC}$ and scheduling algorithms.

The basic CAC algorithm works as follow. When a new connection request arrives, the basic algorithm considers maximum sustained rate (MSR) of that connection as an admission criterion for UGS/ertPS and average rate as an admission criterion for rtPS/nrtPS. If the available bandwidth $\left(B W_{a v_{C A C}}\right)$ is greater than the admission criterion bandwidth $\left(B W_{a c}\right)$, then that connection will be admitted by guaranteeing $B W_{a c}$, otherwise will be rejected. The $B W_{a v_{C A C}}$ is updated after admitting a connection as per equation 1 . The $\mathrm{BE}$ connections are admitted directly since they do not require QoS guarantee.

$$
\begin{aligned}
B W_{a v_{C A C}} & =B W_{a v_{C A C}}-B W_{a c} \\
B W_{\text {guar }} & =B W_{a c}
\end{aligned}
$$

The basic algorithm admits the rtPS connections by guaranteeing average rate even though the network is less congested. This makes the utilization of bandwidth less efficient and leads to the wastage of resources. Also if low priority connections are already admitted and if there is no enough bandwidth to admit the high priority UGS/ertPS connection request then the basic algorithm rejects the high priority UGS/ertPS connection.

In order to overcome the above mentioned drawbacks of basic algorithm, the proposed CAC algorithm tries to improve the utilization of bandwidth efficiently by providing higher bandwidth than the $B W_{a c}$ to the rtPS connection when the network is less congested. Also an attempt has been made to improve the admission of higher priority connections when there is no sufficient bandwidth to admit the request, by degrading the lower priority connections without affecting their QoS performance based on the modulation scheme adopted by SSs.

When a new connection request belonging to UGS/ertPS seeks for admission, the proposed algorithm also considers MSR as an admission criterion as that of basic algorithm. When the network is less congested, the criterion for admission considered for rtPS is $80 \%$ of MSR and for nrtPS is average rate. When the network is congested, the admission criterion for rtPS is average rate and for nrtPS is minimum reserved rate (MRR). If the $B W_{a v_{C A C}}$ is greater than the $B W_{a c}$, that connection will be admitted by guaranteeing $B W_{a c}$ and $B W_{a v_{C A C}}$ is updated as per the equation 1 . If the connection request is for a real time service and $B W_{a v_{C A C}}$ is less than the $B W_{a c}$, then the proposed algorithm tries to admit that connection by degrading the lower priority connections. The additional bandwidth required $\left(B W_{A R}\right)$ to admit the higher priority connection request is calculated using equation 3 .

$$
B W_{A R}=B W_{a c}-B W_{a v_{C A C}} \quad 3
$$

In order to avail the $B W_{A R}$ to admit the real time services, initially nrtPS connections are considered for degradation on the basis of modulation scheme used by the SSs, i.e., connections of SSs with higher order modulation are considered first and then connections of SSs with lower order modulation schemes.

One of the nrtPS connections of SSs which uses 64QAM modulation scheme is selected for degradation from the list of connected SSs. Since 64QAM provides the higher data rate and is 
International Journal of Wireless \& Mobile Networks (IJWMN) Vol. 7, No. 1, February 2015

capable to suffice the QoS requirements of nrtPS connection even with the MRR bandwidth guarantee.

The maximum possible bandwidth $\left(B W_{d e g_{\max }}\right)$ that can be degraded from the nrtPS connection considered is calculated using the equation 4 ,

$$
B W_{\operatorname{deg}_{\max }}=B W_{\text {guar }_{(n r t P S)}}-B W_{M R R_{(n r t P S)}} 4
$$

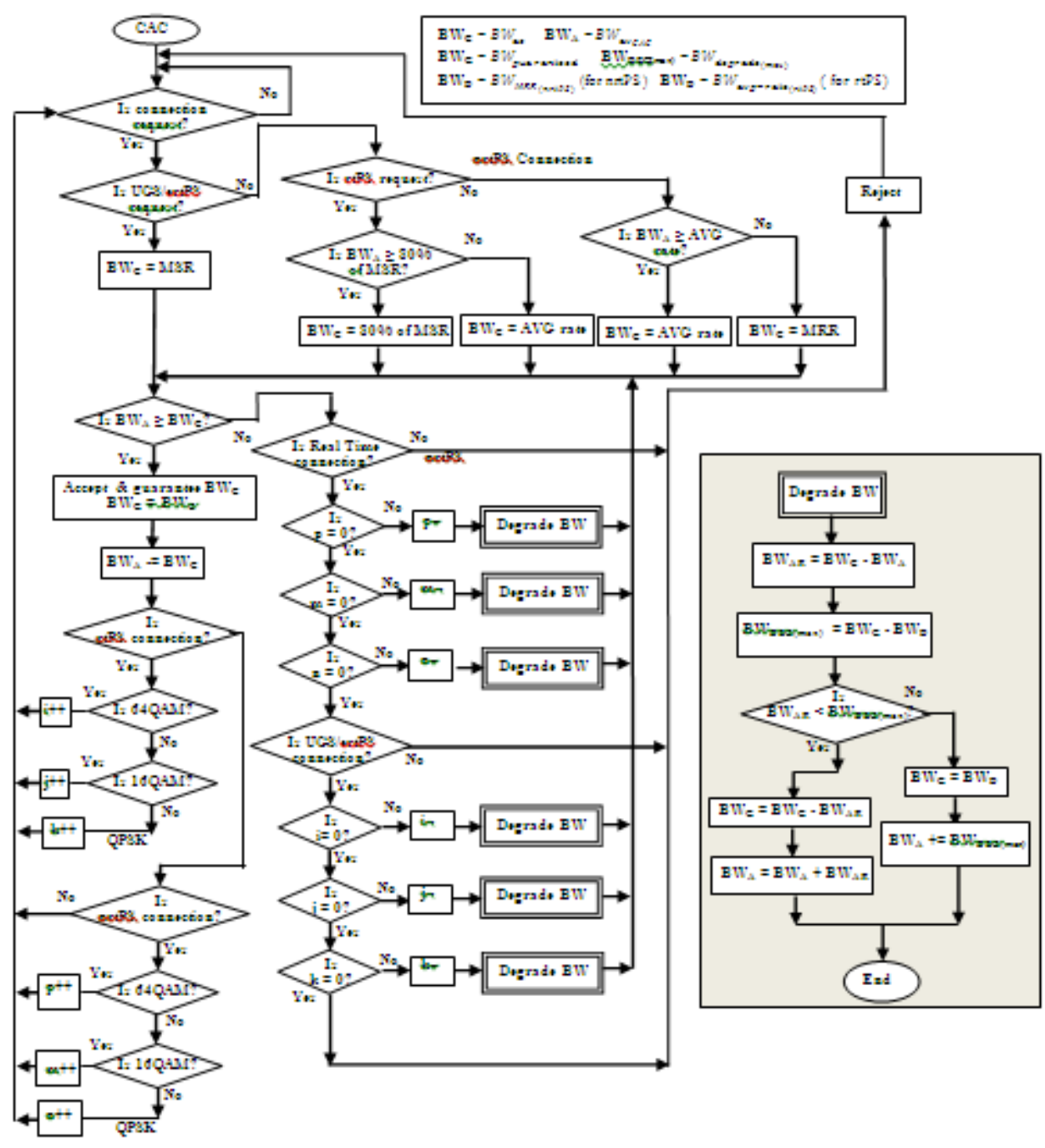

Figure 1. Flow chart of proposed CAC mechanism

If $B W_{d e g_{\max }}$ is greater than the $B W_{A R}$, then bandwidth of nrtPS connection is degraded as per the equation 5 and the requested connection is admitted. The $B W_{a v_{C A C}}$ is updated as per the equation 6.

$$
\begin{aligned}
& B W_{\text {guar }_{(n r t P S)}}=B W_{\text {guar }_{(n r t P S)}}-B W_{A R} \\
& B W_{a v_{C A C}}=B W_{a v_{C A C}}+B W_{A R}
\end{aligned}
$$


If $B W_{d e g_{\max }}$ is less than the $B W_{A R}$, then bandwidth of nrtPS connection considered for degradation is degraded to MRR. The $B W_{a v_{C A C}}$ and $B W_{A R}$ are updated as per the equation 7 and 8 respectively.

$$
\begin{gathered}
B W_{a v_{C A C}}=B W_{a v_{C A C}}+B W_{d e g_{\max }} \\
B W_{A R}=B W_{A R}-B W_{d e g_{\max }}
\end{gathered}
$$

Further to avail the $B W_{A R}$ to admit the real time services, other nrtPS connections of SSs which uses 64QAM modulation scheme are considered for degradation one by one and still the available bandwidth is not sufficient to admit the real time connection request, then nrtPS connections of SSs which uses 16QAM and QPSK modulation scheme are considered for degradation based on the order of modulation one by one.

Further if the bandwidth available is not sufficient to admit the requested real time connection and the request is for UGS/ertPS, to avail the $B W_{A R}$ the rtPS connections are considered for degradation. The guaranteed bandwidth of rtPS connections is degraded till average rate following the same degradation procedure as that of the nrtPS connection. If the available bandwidth after degrading nrtPS and rtPS connections is not sufficient to admit the higher priority connection request that connection request will be rejected. The flow chart of proposed CAC mechanism is shown in figure 1 .

\section{Proposed Modulation Aware Uplink Scheduling Mechanism}

In this paper along with CAC algorithm a modulation aware BS uplink scheduling algorithm is proposed to improve the QoS performances of ertPS, rtPS and BE services. The proposed scheduling algorithm tries to improve the performance of ertPS and rtPS services by making an effort to allocate the requested bandwidth when requested bandwidth is more than the guaranteed bandwidth. Also the proposed algorithm attempts to improve the performance of BE services by allocating the requested bandwidth to the $\mathrm{BE}$ services in a Round-robin way by considering the modulation schemes used by the SSs.

In the basic scheduling algorithm, the bandwidth is allocated to the admitted connections on the basis of service type priority of the connections. That is, bandwidth is allocated to all the UGS connections first, then to ertPS connections, later to the requested rtPS, nrtPS and BE connections. Weighted fair queue (WFQ) scheduling algorithm is used to schedule the connections of same service type. For UGS connections the BS allocates the MSR and for nonUGS connections BS allocates the bandwidth based on the requested bandwidth $\left(B W_{\text {req }}\right)$. If the $B W_{r e q}$ of a connection is less than the guaranteed bandwidth $\left(B W_{\text {guar }}\right)$ of that connection, then BS allocates requested bandwidth for that connection, otherwise that connection is allocated with the guaranteed bandwidth. The available bandwidth $\left(B W_{a v}\right)$ is updated after allocating the bandwidth to each connection as per the equation 9 .

$$
B W_{a v}=B W_{a v}-B W_{\text {allocated }}
$$

The proposed algorithm follows same procedure as that of the basic algorithm for allocating the bandwidth to the connections of all service types, except for ertPS and rtPS connections when requested bandwidth is more than the guaranteed bandwidth.

When requested bandwidth of ertPS/rtPS connections is more than the guaranteed bandwidth, the proposed algorithm degrades the nrtPS connections for which requested bandwidth is less than 
the guaranteed bandwidth. To allocate the requested bandwidth for ertPS/rtPS connections, additional bandwidth required $\left(B W_{A R}\right)$ is calculated using the equation 10 .

$$
B W_{A R}=B W_{\text {req }}-B W_{\text {guar }}
$$
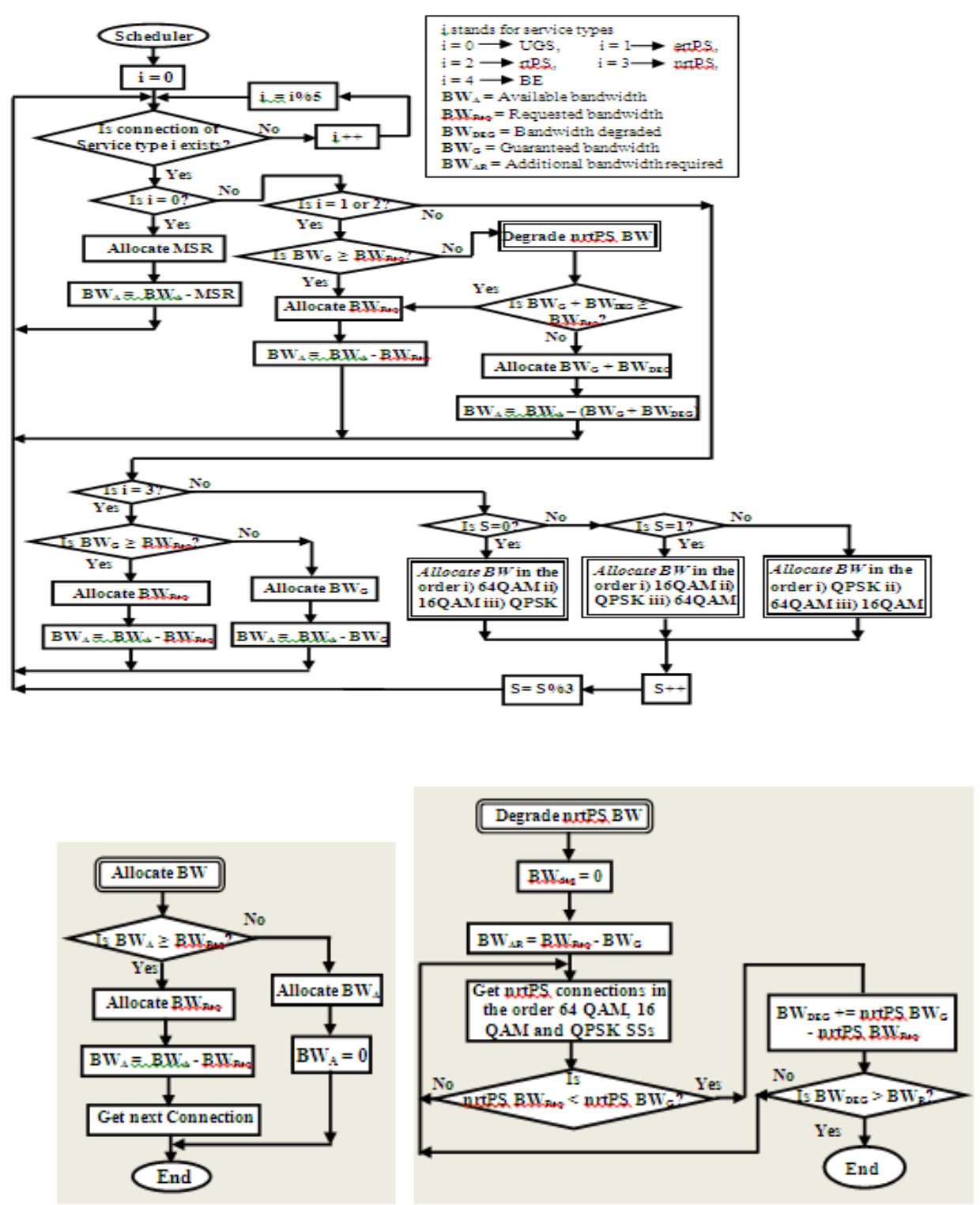

Figure 2. Flow chart of proposed scheduling algorithm

In order to avail $B W_{A R}$, one of the nrtPS connections of SSs which use 64QAM modulation scheme is considered from the list of connected SSs for degradation first. If the requested bandwidth by that nrtPS connection is less than the guaranteed bandwidth for that connection, then the bandwidth is taken out in that frame. 
The additional bandwidth obtained $B W_{\text {additional }}$ and the $B W_{A R}$ are updated as per the equation 11 and 12 respectively.

$$
\begin{gathered}
B W_{\text {additional }}=B W_{\text {guar }_{(n r t P S)}}-B W_{\text {req }} q_{(n r t P S)} \\
B W_{A R}=B W_{A R}-B W_{\text {additional }}
\end{gathered}
$$

Further if the $B W_{A R}>0$, then to avail $B W_{A R}$, other nrtPS connections of SSs which uses 64QAM modulation scheme are considered for degradation and further if $B W_{A R}>0$, then nrtPS connections of SSs which uses 16QAM and QPSK modulation scheme are considered based on the order of modulation one by one. After degrading all the nrtPS connections, if still $B W_{A R}>0$, no further degradations will be carried out. The additional bandwidth ( $B W_{\text {additional }}$ ) obtained by the degradation process is allotted to the considered ertPS/rtPS connection along with the guaranteed bandwidth. Meanwhile, after each degradation process if $B W_{A R} \leq 0$, then ertPS/rtPS connection is allotted with the requested bandwidth.

Further in the proposed algorithm, the BE connections are served in a round robin manner based on the modulation schemes used. In the first frame, one of the BE connections of SSs which uses 64QAM modulation scheme is considered based on the WFQ algorithm for bandwidth allocation. If $B W_{a v}>B W_{r e q}$ of the considered connection, then requested bandwidth is allotted to that connection, otherwise available bandwidth is allotted.

If the bandwidth is available after allocation to considered $\mathrm{BE}$ connection, then other $\mathrm{BE}$ connections of SSs which uses 64QAM modulation scheme are considered for bandwidth allocation one by one in the similar procedure. If the bandwidth is still available, then BE connections of SSs which uses 16QAM and QPSK modulation schemes are considered for bandwidth allocation in the similar procedure till available bandwidth exhaust.

In the second frame, similar procedure is followed to allocate bandwidth to the BE connections. But the BE connections of SSs considered for bandwidth allocation is in the order 16QAM, QPSK and 64QAM. In the third frame, similar procedure is followed to allocate the bandwidth by considering the BE connections of SSs in the order of QPSK, 64QAM and 16QAM modulation schemes.

Further, in the subsequent frames similar procedure is followed to allocate the bandwidth to the BE connections by considering the modulation scheme used by SSs in Round Robin procedure as explained. In this way all the BE connections are served by giving equal priority. The figure 2 gives the flow charts of proposed scheduling algorithm.

\section{SimUlation AND RESUltS}

The performance of proposed channel aware CAC and BS scheduling algorithm is evaluated through simulation studies using QualNet 5.0.2 simulator [19]. The performance of proposed algorithm is compared with the basic algorithm present in simulator. A single WiMAX cell is considered in the simulation area of $2 \mathrm{Km} \mathrm{X} 2 \mathrm{Km}$ working at a frequency $2.4 \mathrm{GHz}$. The path loss model selected is two-ray with constant shadowing model of shadowing mean $4 \mathrm{~dB}$. The traffic generated for various service types along with QoS parameters (MSR and MRR) considered are given in table 1. 
Table 1. Traffic considered for simulation.

\begin{tabular}{|l|l|l|l|l|}
\hline Service Type & Type of Traffic & Data Rate & MSR & MRR \\
\hline UGS & CBR & 64000 & 64000 & 64000 \\
\hline ertPS & VBR & 64000 & 64000 & 16000 \\
\hline rtPS & VBR & 256000 & 256000 & 64000 \\
\hline nrtPS & VBR & 256000 & 256000 & 64000 \\
\hline BE & FTP & & 1000000 & 0 \\
\hline
\end{tabular}

\subsection{Scenario 1}

This scenario is designed to study the performance of implemented work for different channel conditions (64QAM, 16QAM and QPSK region). In this scenario, six SSs are placed in each region, hence total number of SSs considered is eighteen. The SSs have to transmit five traffics each mapped to UGS, ertPS, rtPS, nrtPS and BE respectively. Hence the total number of connections can be 90 , among which each service type has 18 connections. The snapshot of scenario is shown in figure 3 . The simulation studies have been carried out by considering the generation of these traffics in four different schemes for both basic and proposed algorithms. The performances of basic and proposed algorithms are compared by considering the metrics like number of connections admitted, throughput and delay.

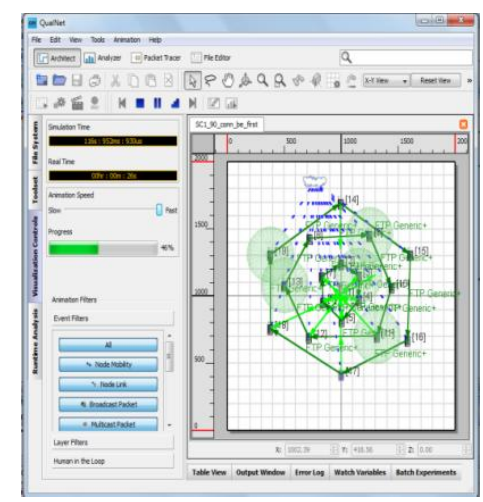

\subsubsection{Scheme 1}

Figure 3. Snapshot of scenario 1

Simulation study has been carried out by considering the generation of traffic on the priority basis from highest priority to least priority, i.e., from UGS to BE. The simulator has been programmed to generate considered traffics at various simulation times as given in the table 2 .

Table 2. Traffic generated and Number of connections admitted for scheme 1.

\begin{tabular}{|c|c|c|c|c|}
\hline $\begin{array}{c}\text { Simulation } \\
\text { time (second) }\end{array}$ & $\begin{array}{c}\text { Number of connections } \\
\text { generated }\end{array}$ & $\begin{array}{c}\text { Service } \\
\text { type }\end{array}$ & \multicolumn{2}{|c|}{ No. of Connections admitted } \\
\hline 1 & 18 & Basic & Proposed \\
\hline 30 & 18 & ertPS & 18 & 18 \\
\hline 60 & 18 & rtPS & 18 & 18 \\
\hline 90 & 18 & nrtPS & 17 & 18 \\
\hline 120 & 18 & BE & 0 & 17 \\
\hline
\end{tabular}


The table 2 and Figure 4 depict the number of connections of different service types admitted by basic and proposed algorithms for scheme 1. It is observed from the table 3 and figure 4 that the number of connections admitted by basic and proposed algorithms is same for scheme 1, since in this scheme, as the higher priority connections are seeking for admission prior to the low priority connections, there is no chance for degradation of low priority admitted connections. It is also observed from the table 3 and figure 4 that the number of $\mathrm{BE}$ connections served is zero since $\mathrm{BE}$ connections have least priority and all the available bandwidth may be utilized to higher priority connections.

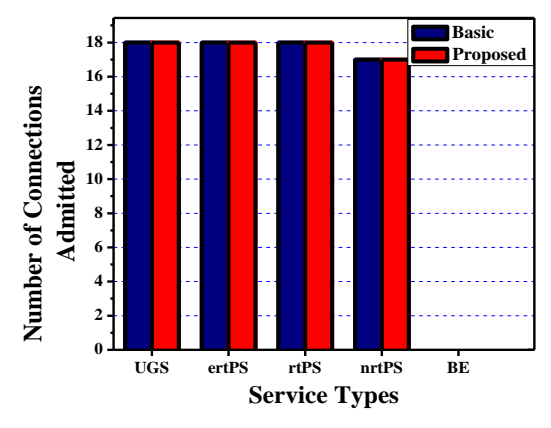

Figure 4. Number of connections admitted - scheme 1

As both basic and proposed algorithms perform in similar manner for scheme 1, the throughput performance is same for all the service types. Hence the throughput performance is not considered here for scheme 1 .

\subsubsection{Scheme 2 and Scheme 3}

Scheme 2: In this scheme, the simulation study has been repeated by generating all the 90 traffics (UGS, ertPS, rtPS, nrtPS and BE connections of 18 each) simultaneously at the beginning of simulation.

Scheme 3: In this scheme the simulation study has been repeated by considering the random generation of traffic. Connections from each service types of one each are generated at every interval of 5 seconds.

Table 3. Number of connections admitted - Scheme 2 and Scheme 3.

\begin{tabular}{|c|c|c|c|c|}
\hline \multirow{2}{*}{$\begin{array}{c}\text { Service } \\
\text { Types }\end{array}$} & \multicolumn{3}{|c|}{ Number of Connections admitted } \\
\cline { 2 - 5 } & \multicolumn{2}{|c|}{ Scheme 2 } & \multicolumn{2}{c|}{ Scheme 3 } \\
\cline { 2 - 5 } & Basic & Proposed & Basic & Proposed \\
\hline UGS & 13 & 15 & 14 & 16 \\
\hline ertPS & 14 & 15 & 15 & 15 \\
\hline rtPS & 13 & 15 & 14 & 15 \\
\hline nrtPS & 13 & 14 & 14 & 15 \\
\hline BE & 5 & 7 & 9 & 8 \\
\hline
\end{tabular}



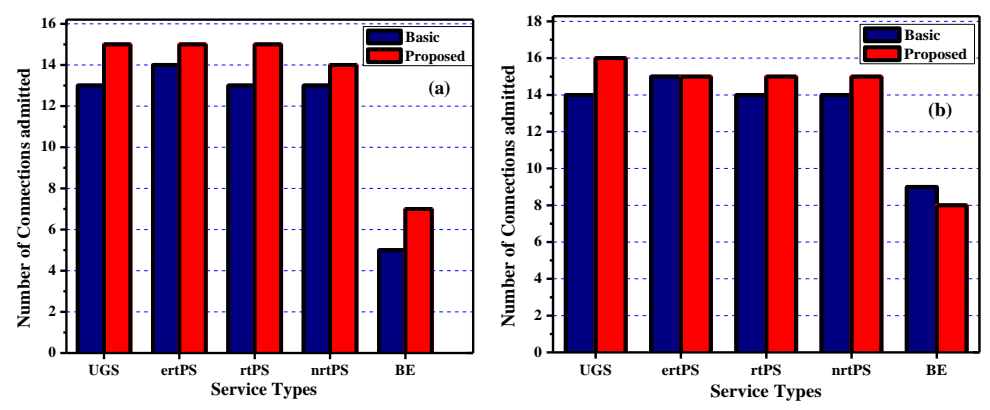

Figure 5. Number of connections admitted for (a) scheme 2 (b) scheme 3

The table 3 and Figure 5(a-b) depict the number of connections admitted by basic and proposed algorithms for scheme 2 and scheme 3 . It is observed from table 3 and figure $5(\mathrm{a}-\mathrm{b})$ that the number of connections admitted in proposed algorithm is more compared to the basic algorithm.
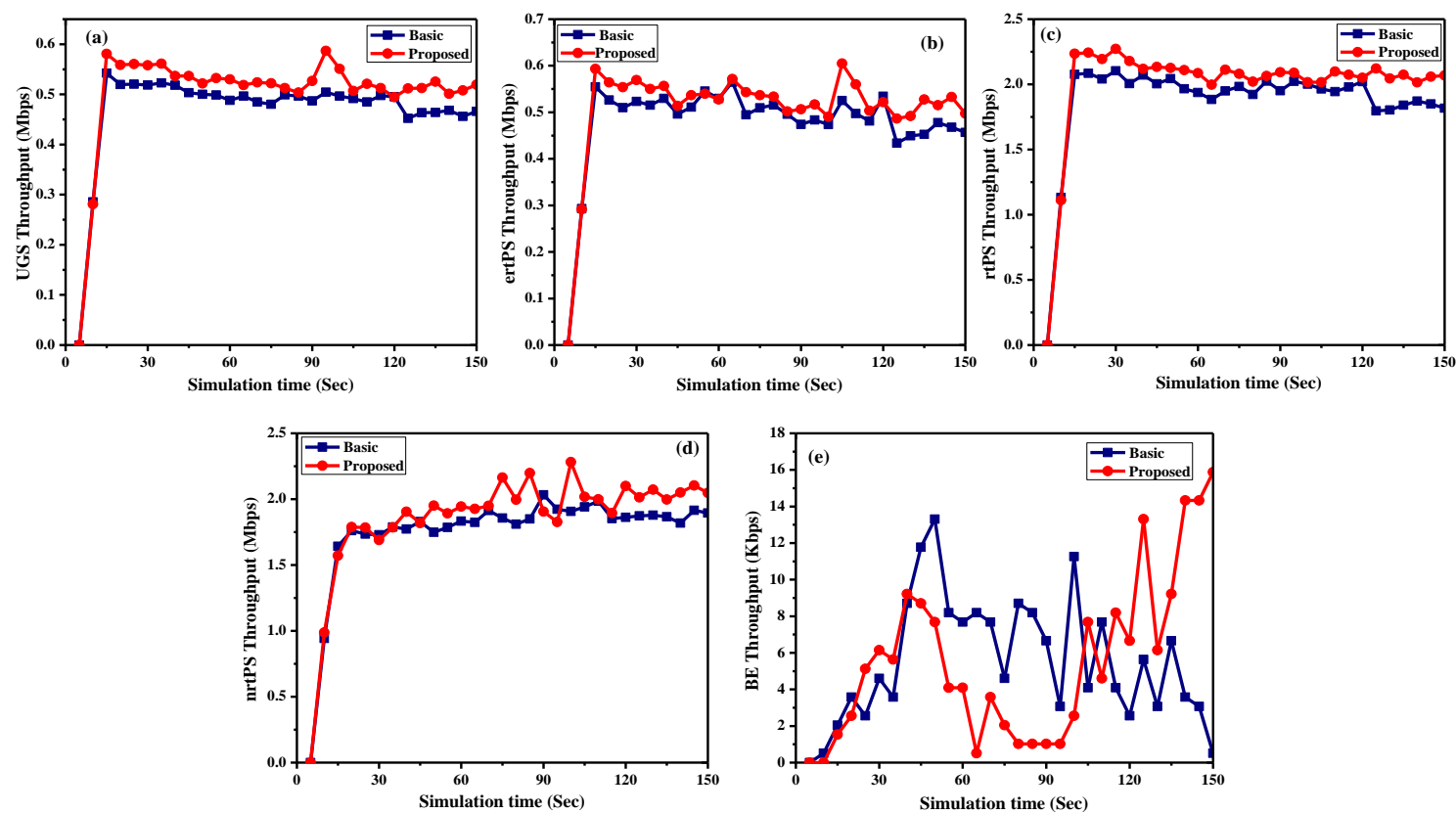

Figure 6. Throughput performance of Scheme -2 for (a) UGS (b) ertPS (c) rtPS (d) nrtPS (e) BE connections
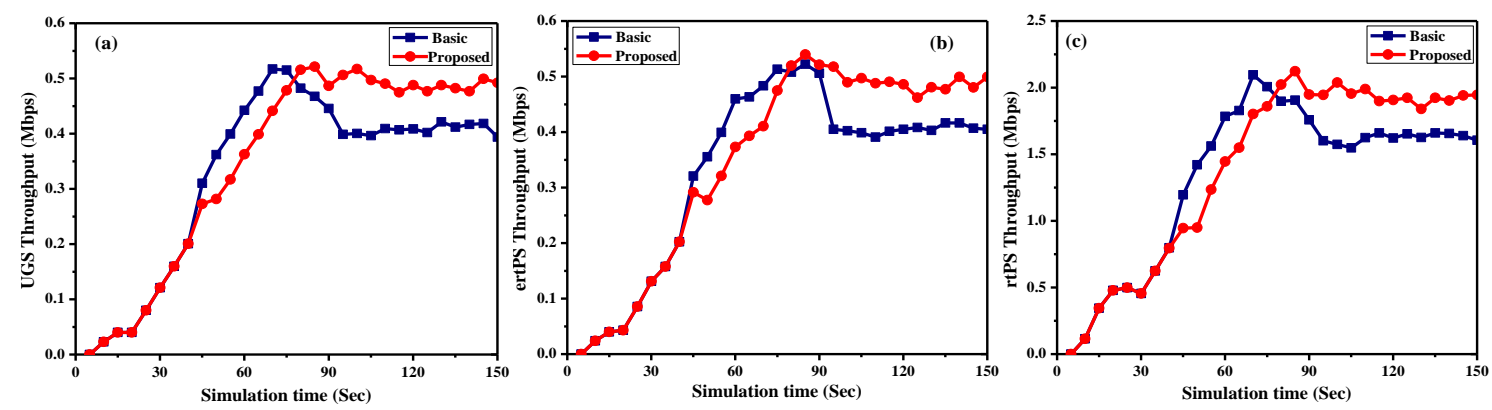

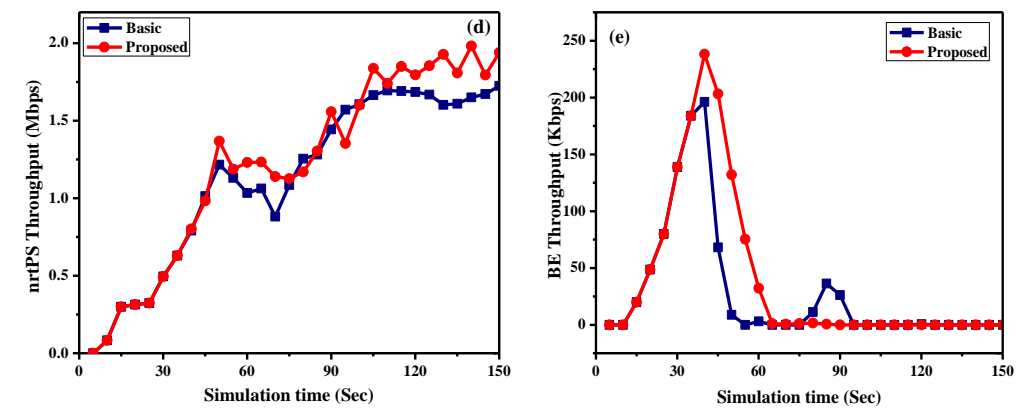

Figure 7. Throughput performance of Scheme - 3 for (a) UGS (b) ertPS (c) rtPS (d) nrtPS (e) BE connections

Figures 6(a-e) and 7(a-e) give the throughput performance of connections of different service types with respect to simulation time for scheme 2 and scheme 3 respectively. It is observed from the figures $6(\mathrm{a}-\mathrm{d})$ and $7(\mathrm{a}-\mathrm{d})$ that the throughput performance of UGS, ertPS, rtPS and nrtPS connections for proposed algorithm is better compared to basic algorithm since the number of connections admitted in proposed algorithm is more compared to basic algorithm. It is observed from the figures 6(e) and 7(e) that the throughput performance of BE connections for proposed algorithm is marginally better compared to basic algorithm.

\subsubsection{Scheme 4}

In this scheme the simulation study has been repeated by considering the generation of traffic in reverse order compared to scheme 1, i.e., the connections are generated from low priority (BE) to high priority (UGS). The traffics generated at various simulation times are given in table 4 .

Table 4. Traffic generated and number of connections admitted for scheme 4.

\begin{tabular}{|c|c|c|c|c|}
\hline $\begin{array}{c}\text { Simulation } \\
\text { time (second) }\end{array}$ & $\begin{array}{c}\text { Number of connections } \\
\text { generated }\end{array}$ & Service type & \multicolumn{2}{|c|}{ No. of Connections admitted } \\
\cline { 3 - 5 } & 18 & BE & 0 & Basic \\
\hline 1 & 18 & nrtPS & 0 & 14 \\
\hline 30 & 18 & rtPS & 18 & 17 \\
\hline 60 & 18 & ertPS & 18 & 16 \\
\hline 90 & 18 & UGS & 13 & 13 \\
\hline 120 & &
\end{tabular}

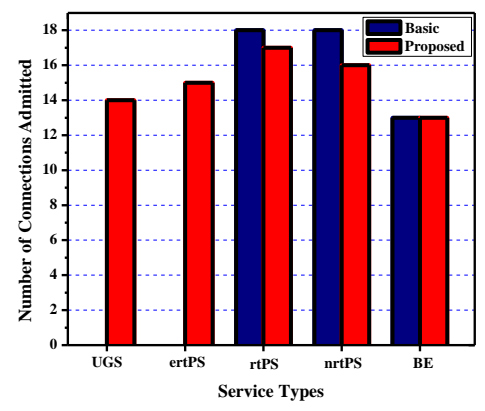

Figure 8. Number of connections admitted - scheme 4

The table 4 and Figure 8 depict the number of connections of different service types admitted by basic and proposed algorithms for scheme 4 . It is observed from table 4 and figure 8 that the 
number of connections admitted in proposed algorithm is more compared to the basic algorithm. The table 4 and figure 8 depict that for scheme 4 the UGS and ertPS connections are not admitted in basic algorithm, whereas the proposed algorithm successfully admits 14 UGS and 15 ertPS connections. The proposed algorithm performs better for scheme 4 since in this scheme the chance to degrade the low priority connections is more than for any other schemes.

Figures 9(a-e) give the throughput performance of connections of different service types with respect to simulation time for scheme 4. It is observed from figure 9(a) that UGS throughput is zero till $120^{\text {th }}$ second for both basic and proposed algorithm, as the UGS connections are started at $120^{\text {th }}$ second. After $120^{\text {th }}$ second the throughput of UGS connections for proposed algorithm increases but the throughput of basic algorithm remains zero. Similarly it is observed from figure 9(b) that ertPS throughput is zero till $90^{\text {th }}$ second, and after $90^{\text {th }}$ second the throughput of ertPS connections for proposed algorithm increases but the throughput of basic algorithm remains zero. As in this scheme the nrtPS and rtPS connections of higher datarate are started before UGS and ertPS, in basic algorithm almost all of the resources of admission control are utilized for admitting the nrtPS and rtPS connections, so the higher priority UGS and ertPS connections are not admitted. Whereas in proposed algorithm, UGS and ertPS connections are admitted since the BS degrades the lower priority connections in order to admit higher priority connections.

It is observed from figure 9(c) and 9(d) that the throughput of rtPS and nrtPS connections for proposed algorithm is less than the basic algorithm, because higher priority connections are get admitted by degrading lower priority connections in proposed algorithm. It is observed from figure 9(e) that throughput performance of $\mathrm{BE}$ connections for proposed algorithm is marginally better compared to basic algorithm.
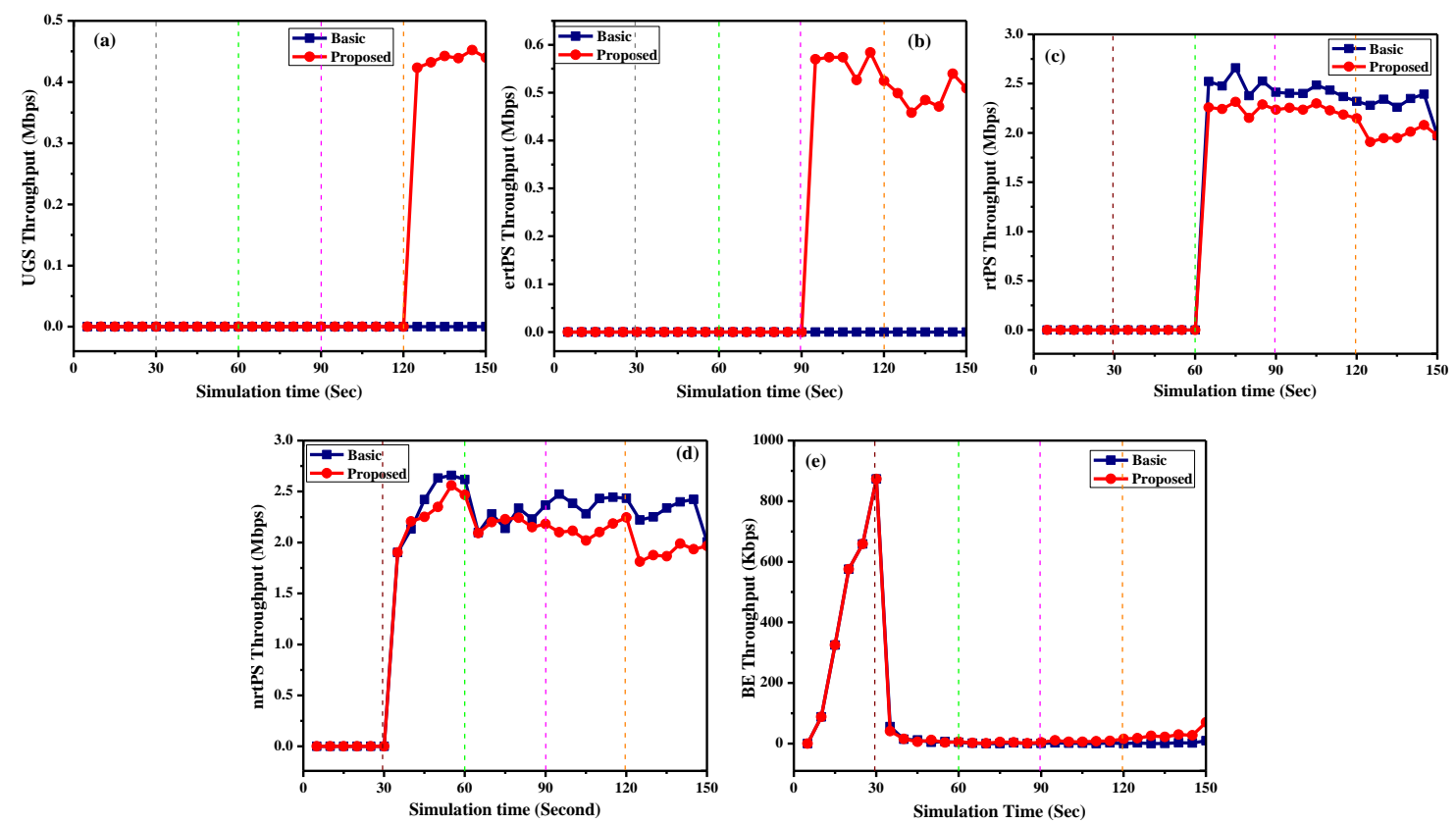

Figure 9. Throughput performance of Scheme - 3 for (a) UGS (b) ertPS (c) rtPS (d) nrtPS (e) BE

\subsection{Scenario 2}

connections

In this scenario, design of scenario 1 is retained and the performance of basic and proposed algorithm are studied by considering number of connections admitted, throughput and delay as 
performance metrics for varying number of connections. Initially 15 connections are considered for study among which 5 connections are considered in each channel conditions (i.e., 5 connections each at 64QAM, 16QAM and QPSK region). Five connections of each region are mapped to UGS, ertPS, rtPS, nrtPS and BE service types. The simulation study has been carried out by generating all the traffics simultaneously at the beginning of simulation. The simulation studies are repeated by increasing the number of connections up to 90 connections insteps of 15 .

Table 5 and figure 10 give the number of connections admitted for varying number of connections. It can be observed that for less number of connections (upto 30 connections) the number of connections admitted in both proposed and basic algorithm are same, since for less number of connections the bandwidth requirement may be less than the available bandwidth, hence degradation is not necessary. As the number of connections increases, the proposed algorithm outperforms basic algorithm. From table 4 and figure 10 it is evident that the number of connections admitted in each service type is more for proposed algorithm compared to basic algorithm. It is also noted that the BE connections served is almost same in both proposed and basic algorithm, as the BE connections do not go through admission control.

Table 5. Number of connections admitted for varying number of connections (B-Basic algorithm, $\mathrm{P}$ - Proposed algorithm)

\begin{tabular}{|c|c|c|c|c|c|c|c|c|c|c|c|c|}
\hline \multirow{2}{*}{$\begin{array}{c}\text { Total number } \\
\text { of Connections }\end{array}$} & $\begin{array}{c}\text { Total Number } \\
\text { of Connections } \\
\text { admitted }\end{array}$ & \multicolumn{8}{|c|}{ Total Number of Connections admitted in each } \\
generated & & \multicolumn{2}{|c|}{ UGS } & \multicolumn{2}{|c|}{ ertPS } & \multicolumn{2}{|c|}{ rtPS } & \multicolumn{2}{|c|}{ nrtPS } & \multicolumn{2}{|c|}{ BE } \\
\cline { 2 - 14 } & $\mathbf{B}$ & $\mathbf{P}$ & $\mathbf{B}$ & $\mathbf{P}$ & $\mathbf{B}$ & $\mathbf{P}$ & $\mathbf{B}$ & $\mathbf{P}$ & $\mathbf{B}$ & $\mathbf{P}$ & $\mathbf{B}$ & $\mathbf{P}$ \\
\hline $\mathbf{1 5}$ & 15 & 15 & 3 & 3 & 3 & 3 & 3 & 3 & 3 & 3 & 3 & 3 \\
\hline $\mathbf{3 0}$ & 29 & 29 & 6 & 6 & 6 & 6 & 6 & 6 & 6 & 6 & 5 & 5 \\
\hline $\mathbf{4 5}$ & 36 & 42 & 7 & 9 & 7 & 9 & 8 & 9 & 7 & 9 & 6 & 6 \\
\hline $\mathbf{6 0}$ & 43 & 57 & 9 & 12 & 8 & 12 & 9 & 12 & 8 & 12 & 9 & 9 \\
\hline $\mathbf{7 5}$ & 64 & 70 & 13 & 15 & 14 & 15 & 14 & 15 & 13 & 15 & 10 & 10 \\
\hline $\mathbf{9 0}$ & 70 & 83 & 14 & 18 & 14 & 18 & 15 & 18 & 13 & 18 & 14 & 12 \\
\hline
\end{tabular}

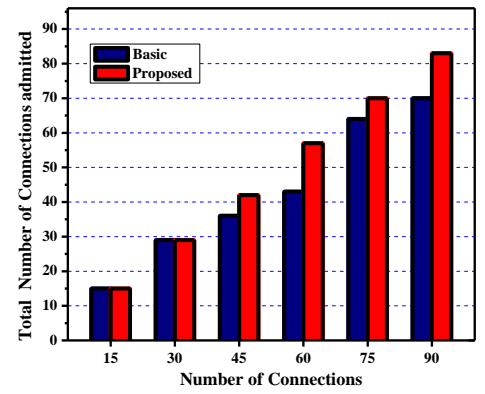

Figure 10. Number of connections admitted for varying number of connections 

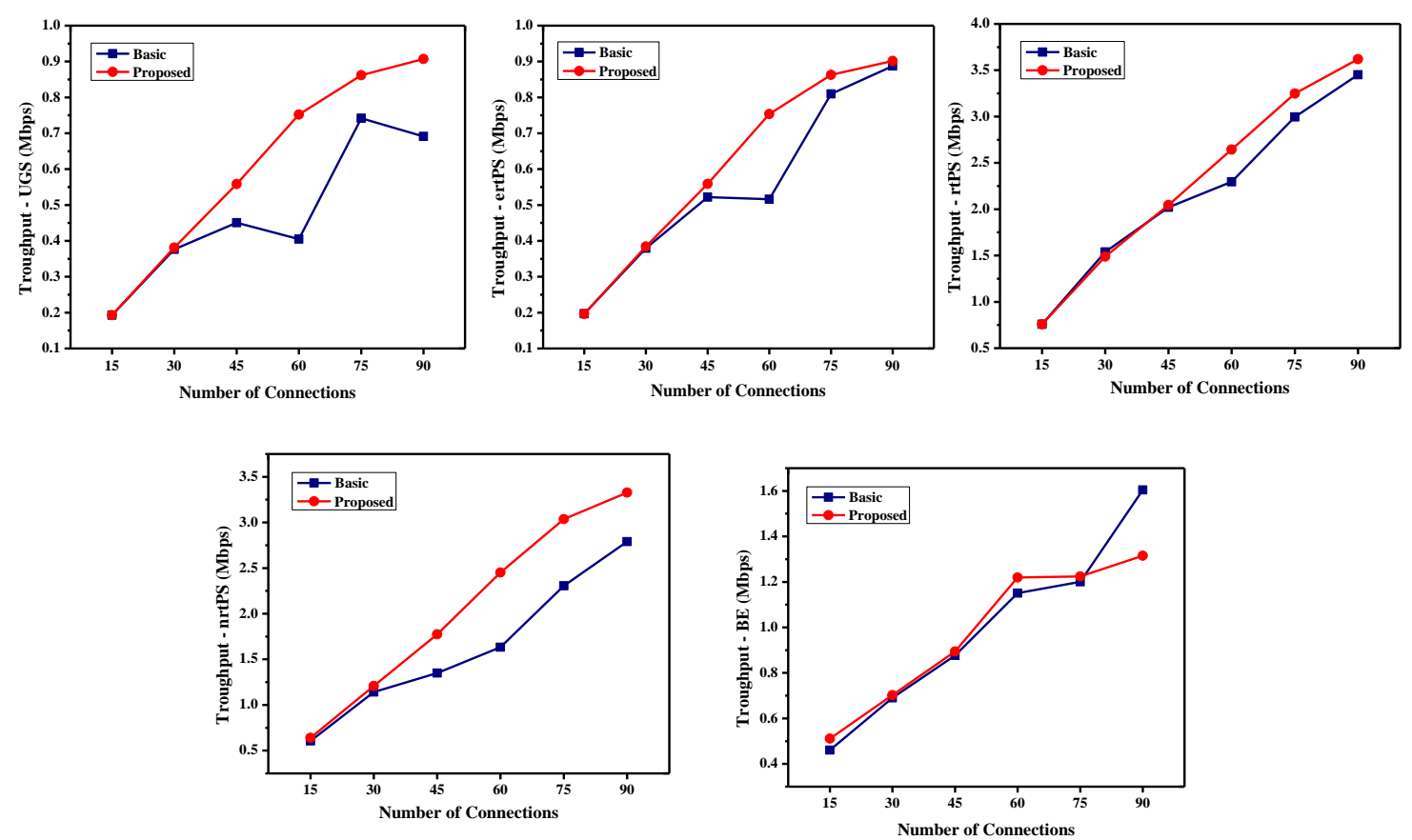

Figure 11. Throughput performance of (a) UGS (b) ertPS (c) rtPS (d) rtPS (e) BE connections

Figures 11 (a-e) give the throughput performances of UGS, ertPS, rtPS, nrtPS and BE connections with respect to varying number of connections respectively. It is observed that for less number of connections the throughput performance is same for both proposed and basic algorithm. As the number of connections increases, the throughput performances of proposed algorithm is better compared to basic algorithm, since the number of connections admitted and served are more in proposed algorithm. It is observed from figure 11(e) that for 90 connections the throughput of $\mathrm{BE}$ for basic algorithm is more because the number of $\mathrm{BE}$ connections served is more in basic algorithm.

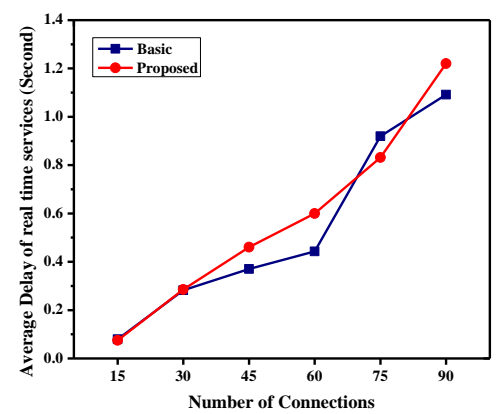

Figure 12. Average delay performance of real time services for varying number of connections

Figure 12 gives the delay performance of real time services for varying number of connections. It is observed that the delay performances are almost same for proposed and basic algorithm.

\subsection{Scenario 3}

This scenario is designed to study the performance of the proposed BS uplink scheduling algorithm for different service types. It is observed from the results of scenario 2 (table 5) that all the generated connections get admitted for the number of connections less than 30 . Hence in this scenario 20 connections are considered for study. The simulation time considered is 100s. 
Initially five connections (one connection from each service type) are considered for simulation and as the simulation progresses the connections of different service types are generated at the interval of three seconds. At $3^{\text {rd }}$ second one UGS connection is generated, at $6^{\text {th }}$ second one ertPS connection is generated and so on. In this way 20 connections (4 connections of each service type) are generated till $45^{\text {th }}$ second. The performance metrics considered for simulation study are throughput and delay. As the proposed scheduling algorithm works in similar way to basic algorithm for UGS and nrtPS cases, the performances of those connections are not considered.
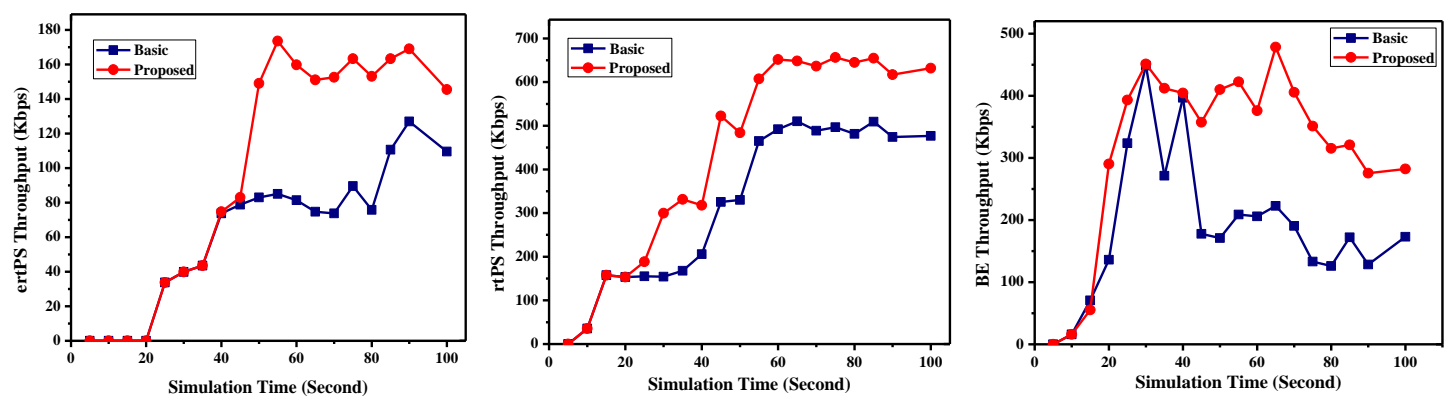

Figure 13. Throughput performance of (a) ertPS (b) rtPS (c) BE connections

Figures 13 (a-c) show the throughput performance of ertPS, rtPS and BE connections respectively with respect to simulation time. It is observed from the figures that throughput increases for both proposed and basic algorithm initially since for every 3 seconds a new connection is get added up. After $50^{\text {th }}$ second the throughput becomes almost constant since no new connections get added after $50^{\text {th }}$ second. Figures also depict that throughput performance of proposed algorithm is better compared to basic algorithm since in proposed algorithm the scheduler degrades the bandwidth guaranteed for nrtPS connections if real time services require more bandwidth, also in proposed algorithm the scheduler serves the BE services in a round robin manner which gives equal priority to all connections of different MCS regions.
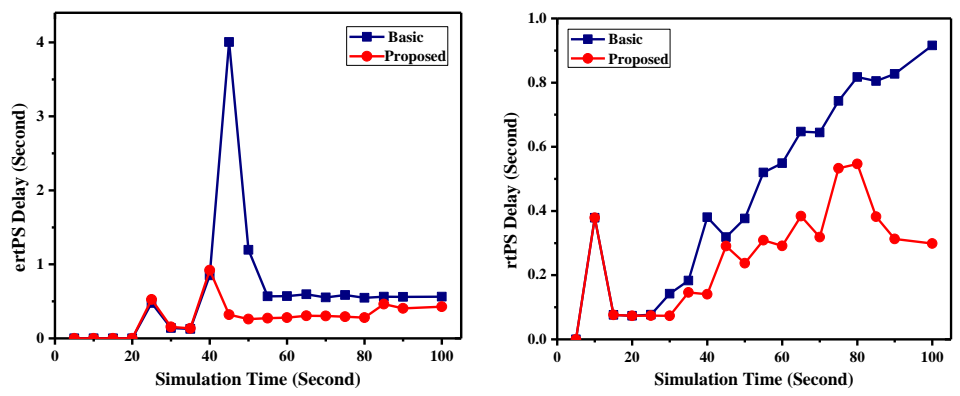

Figure 14. Delay performance of (a) ertPS connections (b) rtPS connections

Figures 14 (a-b) depict the delay performance of ertPS and rtPS connections respectively with respect to simulation time. It is observed from the figures that delay performance of proposed algorithm is better compared to basic algorithm since the proposed algorithm allocates more bandwidth to ertPS and rtPS connections.

\section{Conclusions}

In this paper modulation aware CAC scheme and BS uplink scheduling algorithms are proposed. A connection bandwidth degradation method for lower priority services is proposed to increase 
the system capacity to admit more number of higher priority connections without affecting the QoS performance of lower priority connections much. Also a channel aware BS uplink scheduling algorithm is proposed to improve the QoS performances of ertPS, rtPS and BE services. The proposed algorithms are implemented using QualNet simulation tool. It is observed from the simulation results that the proposed algorithms outperform the basic algorithms.

\section{REFERENCES}

[1] IEEE802.16e: IEEE Standard for Local and metropolitan area networks Part 16: Air Interface for Fixed and Mobile Broadband Wireless Access Systems, 2005.

[2] Byeong Gi Lee and Sunghyun Choi, "Broadband Wireless Access and Local Networks Mobile WiMAX and WiFi", Artech House, 2008.

[3] Haitang Wang, Wei Li and Dharma P. Agrawal, "Dynamic admission control and QoS for 802.16 wireless MAN", Wireless Telecommunications Symposium, 60, 2005.

[4] Kalikivayi Suresh, Iti Saha Misra and Kalpana saha (Roy), "Bandwidth and Delay Guaranteed Call Admission Control Scheme for QOS Provisioning in IEEE 802.16e Mobile WiMAX”, Proceedings of IEEE GLOBECOM, 1245, 2008.

[5] Ke Yu, Xuan Wang, Songlin Sun, Lin Zhang and Xiaofei Wu, "A Statistical Connection Admission Control Mechanism for Multiservice IEEE 802.16 Network”, In Proceedings of VTC Spring, 2009.

[6] R Murali Prasad, "An Efficient Connection Admission Control Mechanism for IEEE 802.16 Networks", Global Journal of Computer Science and Technology, Vol. 10, Issue 8, 97, 2010.

[7] Sarat Chandra and Anirudha Sahoo, "An Efficient Call Admission Control for IEEE 802.16 Networks", In Proceedings of the 15th IEEE Workshop on Local \& Metropolitan Area Networks, 188, 2007.

[8] David A. Levine, Ian F. Akyildiz and Mahmoud Naghshineh, "A Resource Estimation and Call Admission Algorithm for Wireless Multimedia Networks Using the Shadow Cluster Concept", IEEE/ACM Transactions on Networking, Vol. 5, No. 1, 1, 1997.

[9] Lang Xie and Jie Xiang, "A Novel Bandwidth Degradation Scheme for Admission Control in IEEE 802.16e Networks", 4th International Conference on Wireless Communications, Networking and Mobile Computing (WiCOM), 2008.

[10] Romesh Laishram and Iti Saha Misra, "A Bandwidth Efficient Adaptive Call Admission Control Scheme for QoS Provisioning in IEEE 802.16e Mobile Networks", International Journal of Wireless Information Networks, Volume 18 (2), 108, 2011.

[11] Ou Yang and Jianhua Lu, "A New Scheduling and CAC Scheme for Real-Time Video Application in Fixed Wireless Networks”, IEEE CCNC proceedings, 2006.

[12] Samuel K. Falowo and Neco Ventura, "Admission Control and Packet Scheduling for IEEE 802.16 Standards", in proceedings of SATNAC, 2010.

[13] Y. Ge and G.S. Kuo, "An Efficient Admission Control Scheme for Adaptive Multimedia Services in IEEE 802.16e Networks”, IEEE 64th Vehicular Technology Conference (VTC-Fall), 1, 2006.

[14] D. S. Shu'aibu and S. K. Syed Yusof, "Link aware call admission and packet scheduling for best effort and UGS traffics in mobile WiMAX”, International Journal of the Physical Sciences Vol. 6(7), pp. 1694-1701, 2011

[15] Wan Kim and Hwangjun Song, "QoS-aware joint working packet scheduling and call admission control for video streaming service over WiMAX network", Springer - Wireless Networks, 1083, 2011.

[16] Shafaq B. Chaudhry and Ratan K. Guha "Adaptive Connection Admission Control and Packet Scheduling for QoS Provisioning in Mobile WiMAX", IEEE International Conference on Signal Processing and Communications (ICSPC), 2007.

[17] Loutfi Nuaymi, "WiMAX: Technology for Broadband Wireless Access", John Wiley \& Sons Ltd, 2007.

[18] Kwang-Cheng Chen and J. Roberto B. de Marca, "Mobile WiMAX", IEEE PRESS, IEEE Communications Society and John Wiley \& Sons Ltd, 2008.

[19] http://www.scalablenetworks.comlqualnetldocumentation Documentation of QualNet simulation tool 